\title{
CHARACTERIZATION OF DIELECTRIC PROPERTIES OF COMMERCIAL CERAMIC CAPACITORS FOR PULSED POWER APPLICATIONS
}

\author{
Silva Neto, L.P. ${ }^{1}$; Rossi, J.O. ${ }^{2}$; Silva Junior, A. R. ${ }^{3}$ \\ National Institute for Space Research, Associated Plasma Laboratory \\ P.O. Box 515, 12245-970, S.J. Campos, SP, Brazil \\ 1'silvaneto007@yahoo.com.br; ${ }^{2}$ rossi931@hotmail.com; ${ }^{3}$ ataidejr2003@yahoo.com.br
}

\begin{abstract}
It is found currently a wide diversity of dielectrics used for the construction of capacitors, where barium and strontium titanate-based ceramics are the most used. This study concerns the dielectric characterization of commercial ceramics capacitors for use in high voltage capacitors of high-energy storage systems and soliton wave generation for RF production in space applications. To characterize the dielectric material two commercial ceramic capacitors were submitted to HV tests to determine the dielectric constant variation with the applied voltage and the dielectric strength voltage (breakdown -BD) under pulsed conditions. The results show that ceramic capacitors present BD strength of the order of 90-200 kV/cm and non linear behavior, i.e. the ceramic dielectric constant is greatly reduced with the voltage increase.
\end{abstract}

Keywords - barium and strontium titanates, ceramic capacitors, dielectric constant, breakdown voltage

\section{INTRODUCTION}

Ceramic dielectrics (barium and strontium titanates) have been used as dielectric mediums in high-energy storage devices (HV capacitors) due to the high dielectric constant and high dielectric strength presented by these materials. The project is primarily focused on the study of ceramic dielectric of commercial ceramic capacitors used in high voltage applications for high-energy storage systems. Due to the characteristics of non-linearity presented by ceramic capacitors, they are also used in the construction of lumped nonlinear transmission lines (NLTLs) for RF soliton generation [1]. NLTLs are good alternatives for compact systems of RF production to be used in space applications (small spacecrafts and satellites) or in mobile defense platforms, without using electronic tubes (such as klystrons) that are of bigger dimensions and require heating filament power.

Barium titanate-based capacitors are built with a mixed doping of strontium and calcium zirconate in order to shift the dielectric Curier temperature below the room temperature $\mathrm{T}_{\mathrm{A}}$ so that the material is in the paraeletric phase providing the largest dielectric constant around $\mathrm{T}_{\mathrm{A}}[2]$. The doping concentration and proportion between strontium and calcium zirconate define a strong or weak dependence of the material dielectric constant with the applied voltage (.i.e. the nonlinear characteristic of the ceramic dielectric). For example, in pulsed power linear applications a weak dependence is aimed as LC pulse forming networks require stable capacitance. However, in nonlinear applications such as in NLTLs for RF generation strong $\varepsilon \times \mathrm{V}$ dependence is desired because of the nonlinearity effect of the line capacitance. Also a strong linear effect is very useful in pulse sharpening circuits [3], where the rapid variation of $\mathrm{C}$ with the pulse amplitude injected into the NLTL leads to the output pulse rise compression at the output in ns range. As NLTLs operate on pulsed conditions other point of the study is the determination of the $\mathrm{BD}$ voltage of the ceramic dielectric material that composes ceramic commercial capacitor. In fact, this is the main scientific contribution of our paper as there is a paucity of this research on pulsed BD voltage of ceramic dielectrics. Normally, it is found in the literature [4-6] the capacitance or dielectric constant of ceramic capacitors, their total impedance $\mathrm{Z}$ and dissipation factor (loss tangent) characterized as a function of frequency, voltage, temperature, etc. The reason is that these characterization tests are very useful for DC, AC and high frequency applications. Other point is that the results presented herein are for high voltage capacitors, which are not thoroughly documented.

\section{EXPERIMENTAL SET-UP}

The experiment used four different commercial capacitors with specifications given in the Tab. 1. For the capacitance measurements we used a HV DC power supply that biased the device under test (DUT) and four HV blocking capacitors $(40 \mu \mathrm{F})$ in series for isolating the meter input from the DC HV voltage bias (see Fig. 1). The blocking capacitance must be at least 10 times greater than the capacitance of the DUT to not cause much interference with the measurement. As the signal amplitude of the capacitance meter is much smaller than the bias DC voltage, the meter reading gives strictly a measure of the differential capacitance for the capacitor under test [7].

TABLE I

Characteristics and Dimensions of the capacitors used

\begin{tabular}{ccclc}
\hline Capacitor & Voltage & Capacitance & Diameter & Thickness \\
\hline 1 & $3 \mathrm{kV}$ & $0.47 \mathrm{nF}$ & $5.08 \mathrm{~mm}$ & $1.18 \mathrm{~mm}$ \\
\hline 2 & $2 \mathrm{kV}$ & $1.0 \mathrm{nF}$ & $5.60 \mathrm{~mm}$ & $0.68 \mathrm{~mm}$ \\
\hline 3 & $1 \mathrm{kV}$ & $4.7 \mathrm{nF}$ & $7.5 \mathrm{~mm}$ & $0.50 \mathrm{~mm}$ \\
\hline 3 & $3 \mathrm{kV}$ & $100 \mathrm{pF}$ & $6.81 \mathrm{~mm}$ & $0.61 \mathrm{~mm}$ \\
\hline
\end{tabular}

The respective relative dielectric constant $\mathrm{k}$ of the capacitor dielectric under the $\mathrm{C} \times \mathrm{V}$ tests is obtained through: 


$$
k=\frac{C d}{\varepsilon_{0} A}
$$

where $\mathrm{C}$ is the measured capacitance, $\mathrm{d}$ is the capacitor dielectric thickness, $\mathrm{A}$ is the capacitor dielectric area and $\varepsilon_{0}$ is the free-space vacuum permittivity.

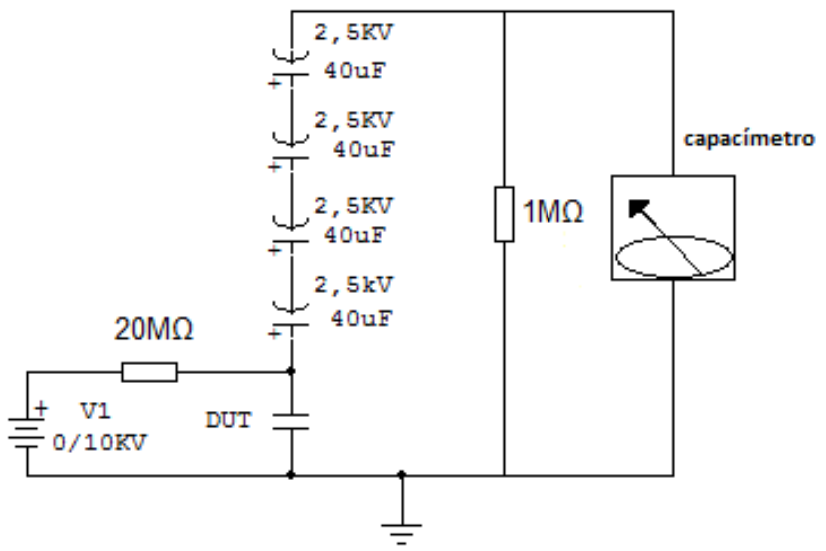

Fig. 1. Circuit used for the $\mathrm{C} \times \mathrm{V}$ measurement on $\mathrm{DC}$ condition.

For the HV BD tests we used an LC oscillatory half sine wave circuit [8] as showed in Fig. 2 (a) \& (b). This circuit is composed of two parts. The first part (a) consist of a DC power supply responsible for charging capacitor bank of 1.5 $\mu \mathrm{F} / 800 \mathrm{~V}$ formed by capacitors $\mathrm{C} 3, \mathrm{C} 4, \mathrm{C} 5$ and $\mathrm{C} 6$ with same specification of $1.5 \mu \mathrm{F} / 400 \mathrm{~V}$. This capacitor bank is discharged via switch S1 through the primaries of two car ignition coils (model Bosch KW 12V) connected in parallel as shown in the second part (b) of the circuit. In order to generate a HV differential pulse on the sample to be tested, the secondary windings of both coils are biased positively and negatively. As described in part (b) of the circuit, reversed diodes connected across both coils are used to suppress the negative voltage excursion on the capacitor bank to limit input current inrush drained from the DC power supply as there is no current control. With full DC charging voltage a differential pulse peak of about $60 \mathrm{kV}$ is applied across the positive $\&$ negative terminals of the pulser. The pulse duration is of the order of $100 \mu$ s and normally the sample may breakdown near the pulse peak, interrupting only the first resonant cycle of the pulse generator and keeping the subsequent ones of lower amplitudes due to the residual voltage across the capacitor bank. During the HV tests, we adjusted the DC power supply of the LC pulser for a maximum voltage of about $300 \mathrm{~V}$, which is equivalent to $50 \%$ of the variac input voltage.

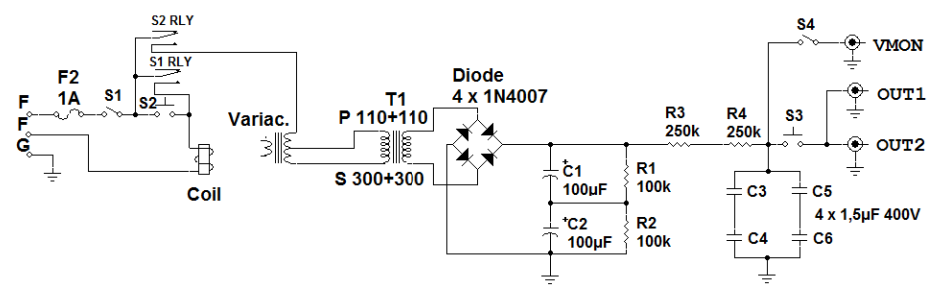

(a)

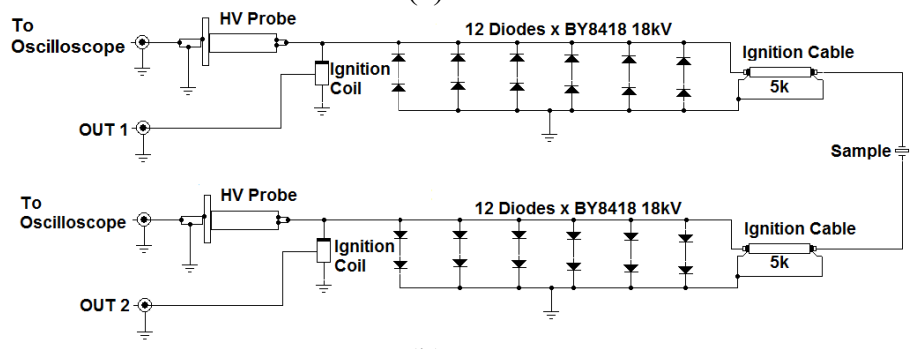

(b)

Fig. 2. The primary circuit presented for input impulse generation (a), and the associated HV output circuit with the ignition coils (b).

\section{RESULTS AND DISCUSSION}

Figs. 3, 4 and 5 show respectively the $\mathrm{C} \times \mathrm{V}$ (in black) and $\mathrm{k} \times \mathrm{V}$ (in blue) measurements on static condition respectively for capacitors 1, 2 and 3 using the circuit of Fig. 1. For capacitor 4 , the $\mathrm{C} \times \mathrm{V}$ curve was not traced as the connection stray capacitance of the meter circuit started to compromise the measurement due to the small capacitance to be measured (less than $400 \mathrm{pF}$ ). Note that for the three cases the $\mathrm{k}$ measurement follows the $\mathrm{C}$ variation and the capacitance decreasing with the voltage applied as expected for the barium titanate based dielectric ceramics. The explanation for this effect is that after an initial alignment the dielectric dipoles reach very fast the saturation point and as the applied field is increased the dipole alignment start to distort causing a reduction in $\mathrm{k}$ as observed [7]. Obviously this phenomenon is not desired for linear applications that require stable capacitance not dependable on the capacitor charging voltage. On the contrary, as mentioned before this effect is intensely explored for nonlinear applications in NLTLs and pulse sharpening circuits.

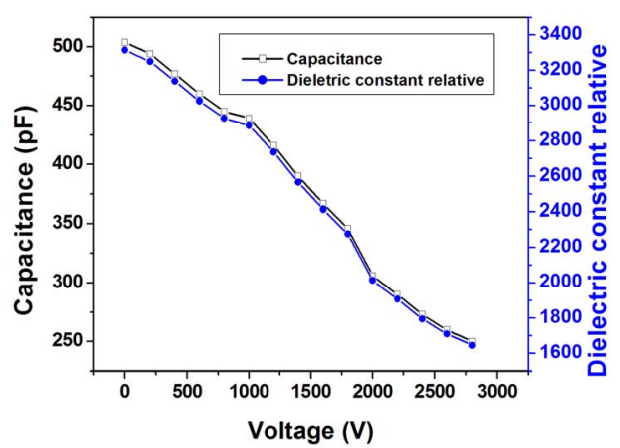

Fig. 3. $\mathrm{C} \times \mathrm{V}$ measurement obtained for capacitor 1 . 


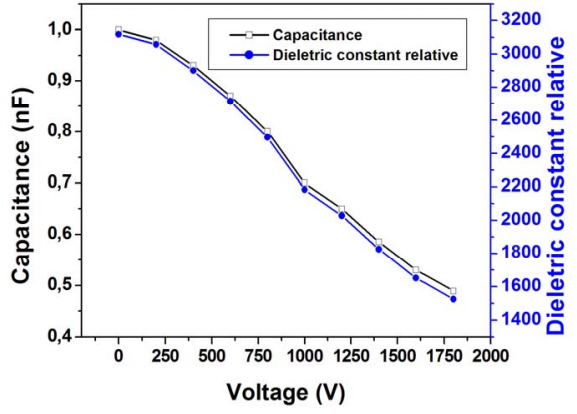

Fig. 4. $\mathrm{C} \times \mathrm{V}$ measurement obtained for capacitor 2 .

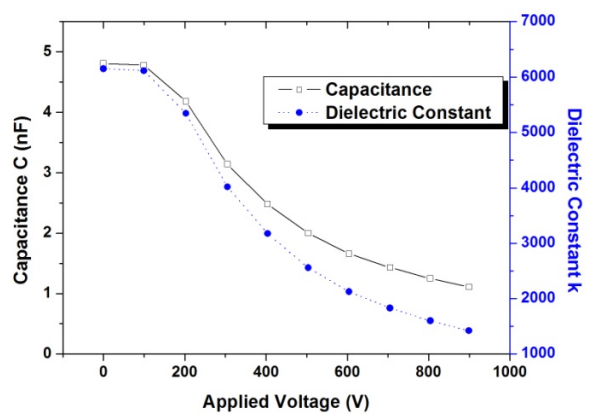

Fig. 5. $\mathrm{C} \times \mathrm{V}$ measurement obtained for capacitor 3 .

Other point investigated was the breakdown voltage of capacitors given in Tab. 1 under pulsed conditions since NLTLs work normally with HV pulses injected at their input with ns or $\mu$ s duration. Figs. 6,7 and 8 show respectively the results obtained from the BD tests for capacitors $1,2 \& 4$ using the circuit of Fig. 2. For capacitor 3, the LC pulse generator was not capable of producing the $\mathrm{BD}$ voltage required due to its bigger capacitance $(>1 \mathrm{nF})$. In this case, the primary voltage of the ignition coil is attenuated sufficiently to limit the differential output voltage to less than $5 \mathrm{kV}$ as the capacitance under test is reflected to the input side with squared coil turns ratio $\left(\mathrm{n}^{2}\right)$. Observe in these figures that $\mathrm{BD}$ voltages are determined considering the point at which both positive \& negative output voltages drop very rapidly to zero during the first half sine wave applied by the LC oscillator. From Fig. 6, one obtains a peak-to-peak BD voltage of approximately $10 \mathrm{kV}$ for capacitor 1 , giving a dielectric strength of about $8.6 \mathrm{kV} / \mathrm{mm}$ for a $1.18 \mathrm{~mm}$ dielectric width (according to Tab. 1). In the case of capacitor 2, from Fig. 7 obtains a BD voltage of about $7 \mathrm{kV}$ which gives a dielectric strength of the order of $10.3 \mathrm{kV} / \mathrm{mm}$ for a dielectric width of $0.68 \mathrm{~mm}$ in Tab. 1. Similarly, for capacitor 3, obtains from Fig. 8 a dielectric strength of the order of $20.3 \mathrm{kV} / \mathrm{mm}$ for a BD voltage of about $12.4 \mathrm{kV}$ with a $0.61 \mathrm{~mm}$ dielectric thickness. From these results, observe that the dielectric BD strength (BDS) increases when the dielectric thickness decreases. As described elsewhere in [9] there is a strong dependence of the BDS of the nanostructure ceramic materials as a function of the dielectric thickness. A reasonable explanation for this is that with reduced thickness the probability of forming voids with air or structure defects in the bulk of dielectric, which causes a reduction in the value of the electric field required to produce the $\mathrm{BD}$, is lower during the ceramic sintering process. Also note that the
BD voltages of capacitors $1,2 \& 4$ under pulses conditions are significantly higher than their respective BD DC voltages by a factor that varies between 3 and 7 approximately, depending on the tested capacitor. This means that the capacitors $(1,2 \& 4)$ tested support pulse amplitudes slightly below their respective pulsed $\mathrm{BD}(10,7$ and $12 \mathrm{kV})$ voltages since at the BD point the dielectric is damaged, as shown in Fig. 9 by the flashover channel formed for capacitor 2 during the BD test with 50x resolution. Consequently, on this condition after the BD tests the components are discarded. On the other hand, for storage systems or in linear lumped lines such as PFNs the nominal working voltages of capacitors are limited up to their DC BD voltages.

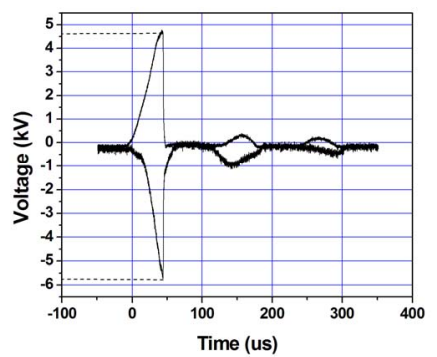

Fig. 6. BD voltage measured of $10 \mathrm{kV}$ for capacitor 1 .

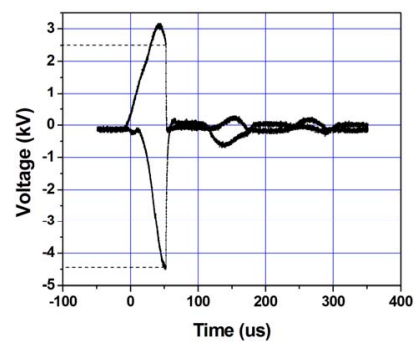

Fig. 7. BD voltage measured of $7 \mathrm{kV}$ for capacitor 2 .

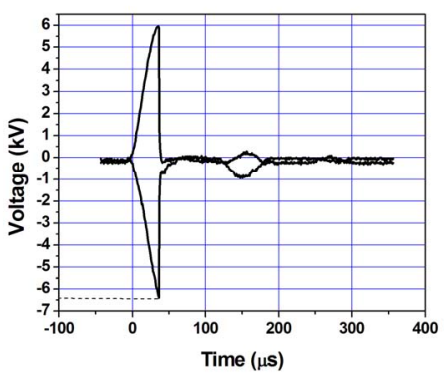

Fig. 8. BD voltage measured of $7 \mathrm{kV}$ for capacitor 4 .

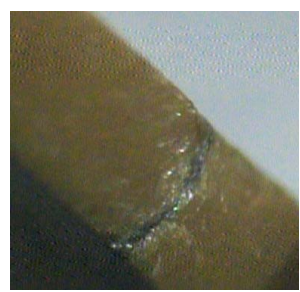

Fig. 9. Flashover channel formed on the capacitor 2 border. 
In Fig. 9, the channel image was obtained with a stereoscopy (Topcon) coupled to a CCD digital camera from Sony, but the same image with better resolution (500x) was also provided by a scanning electron microscope (SEM) given in Fig. 10, where one can see clearly the channel formed across the ceramic bulk as shown by the area delimited by the yellow line. The SEM device employed (Jeol model- JSM 5310) is also equipped with x-rays energy dispersive analyzer (EDS) that gives the chemical composition of the material sample under diagnostic as given in Fig. 11. From this EDS figure, we can see several peaks of Barium and Titanium, which demonstrates that the capacitor dielectric under study is composed basically by a mixture involving basically barium titanate oxides $\left(\mathrm{BaTiO}_{3}\right)$, being responsible for the nonlinear characteristics of the dielectric as evidenced by the $\mathrm{C} \times \mathrm{V}$ material characteristic measured as expected.

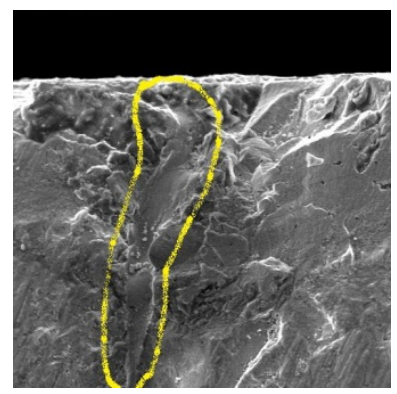

Fig. 10. Flashover channel showed with better resolution.

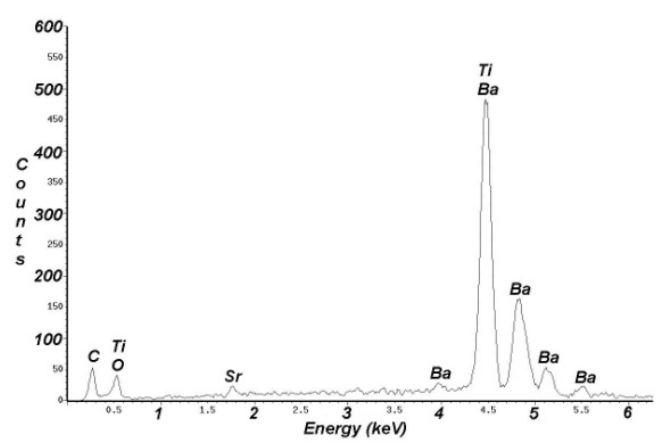

Fig. 11. EDS spectrum obtained for capacitor 2 dielectric.

\section{CONCLUSIONS}

The results showed the ceramic commercial capacitors can be used in linear applications if the system is charged rather below their DC breakdown. This is so because up to half the DC capacitor nominal voltage the capacitance variation is generally less than $20 \%$, which can be acceptable when working with PFNs for rectangular flat pulse generation. Nevertheless, for high-energy storage systems a trade-off between capacitance and operation voltage must be compromised as the energy stored into the dielectric is proportional to $0.5 \times \mathrm{CV}^{2}$. Depending on the nonlinearity characteristic of the dielectric the $\mathrm{C}$ variation can be compensated very easily by the factor of squared V. Other important characteristic investigated is the $\mathrm{BD}$ voltages of the capacitors that revealed that their dielectrics can support pulses with amplitudes of at least 3 times their DC maximum nominal working voltages. This property is of great importance for constructing nonlinear lumped lines with reduced number of capacitors as they support higher BD voltages. For instance, when working with NLTLs for pulse rise time compression in pulse sharpening circuits an LC nonlinear line can be built employing these ceramic commercial nonlinear capacitors. If a HV pulse with a long rise time (hundreds of ns) is injected at the input of the line, an output pulse is obtained with a shorter pulse rise (normally lower than about $100 \mathrm{~ns}$ ), where the rise time pulse reduction is determined approximately by the line capacitance variation during the pulse application. This technique is largely used in circuits that require pulses with higher amplitudes (several $\mathrm{kV}$ ) and output rise time in the range of 10-100 ns and where the use of fast solid sate switches are not recommended because of the high-voltage environment. A more specific application for this case can be found in [10], in which a $20 \mathrm{kV}$ NLTL pulse compression circuit built with $2 \mathrm{kV} / 4.7 \mathrm{nF}$ capacitors from Murata was used to feed a TEA-CO $\mathrm{CO}_{2}$ Laser. They described that the NLTL compressed an input pulse of $20 \mathrm{kV} / 500 \mathrm{~ns}$ into an output pulse of $20 \mathrm{kV} / 120 \mathrm{~ns}$ for laser activation.

Other important application derived from the nonlinearity of ceramic capacitors is surprisingly found in the RF generation using only these nonlinear lumped lines, instead of conventional RF vacuum tubes such as klystron or magnetrons. Considering the fact that the shortest rise-time $t_{s}$ in these lines is limited by the ladder cut-off frequency $\left(\omega_{C}=2 / \sqrt{ }(L C)\right)$ frequency, pulse compression is not possible if a fast pulse with rise time $<t_{s}$ is injected into the line, normally in the sub-ns range. In this case, it is observed high-frequency oscillations superimposed on the pulse, being particularly strong at the middle sections of the lumped line. If a load such as an antenna is placed at the line output RF extraction and irradiation is possible. This new application has been recently the focus of intense research as described in [11] and is aimed for use in very compact RF pulse generation for space vehicles and defense mobile platforms as conventional RF tubes are of bigger size and require filament heating power. At present, with the use of this technique they [11] demonstrate the feasibility of generating $20 \mathrm{MW}$ of RF power peak at $1 \mathrm{GHz}$ with pulse repetition rate of about $1 \mathrm{kHz}$.

\section{ACKNOWLEDGEMENT}

Work sponsored by the USAF SOARD under contract number FA9550-10-1-0013. The author Siva Neto also acknowledges Brazilian funding Agency (CNPq) for supporting him with a graduate scholarship.

\section{REFERENCES}

[1] P.W. Smith, Transient Electronics - Pulsed Circuit Technology, West Sussex, England: John Wiley \& Sons, pp. 245-249, 2002. 
[2] S. Roberts, "Dielectric and Piezoelectric properties of Barium Titanate," Physical Review, vol. 71, no.12, pp.890-895, 1947.

[3] C.R. Wilson et al., Pulse Sharpening in a Uniform LC Ladder Network Containing Nonlinear Ferroelectric Capacitors," IEEE Trans. Electron Dev., vol.38, pp. 767$771,1991$.

[4] D. Burks, R. Hofmaier, S. Knudtsen, and G. Shirn, "A ceramic capacitor for AC applications," in Proc. of the $39^{\text {th }}$ IEEE Electronic Components Conference, 1989, pp. 194-201.

[5] G.H. Maher, "A new PLZT dielectric for use in characteristics X7R multilayer ceramic capacitors," IEEE Trans. on Components, Hybrids, Manufacturing Technology, vol. CMHT-6, no. 4, Dec. 1993, pp. 372376.

[6] J.T. Strydom, J.D. Van Wyk, and J.A. Ferreira, "Capacitor measurements for power electronic applications," in Proc. of the IEEE Industry Applications Conference, 1999, pp. 2435- 2440.

[7] J.O. Rossi; R.H.M. Siqueira, and L.P. Silva, "Study of capacitance dependence of ceramic commercial capacitors on the voltage and temperature," in Proc. of the $19^{\circ}$ CBECiMat, Campos do Jordão, Nov. 2010, pp. 1082-1089.

[8] P. Castro, C.J. Buchenauer, J. Gaudet, and E. Schamiloglu, "Studies of dielectric breakdown under pulsed power conditions," in Proc. of the $15^{\text {th }}$ IEEE Pulsed Power Conf. (Monterey, CA 2005), pp. 978-971.

[9] J.A. Gaudet et al., "Research issues in developing compact pulsed power for high peak power applications on mobile platforms," Proc. of the IEEE, vol. 92, no. 7, July 2004, pp. 1144-1165.

[10] S. Ibuka, K. Abe, T. Miyazawa, A. Ishii, and S. Ishii, "Fast high-voltage pulse generator with nonlinear transmission line for high repetition rate operation", IEEE Transactions on Plasma Science, vol. 25, no.2, April 1997, pp. 266-271.

[11] N. Seddon, C.R. Spikings, and J.E. Dolan, "RF pulse formation in nonlinear transmission lines," Proc. $16^{\text {th }}$ IEEE Int. Pulsed Power Conf. (Albuquerque, NM 2007), pp. 678-681. 